\title{
Activation of Protein Kinase C Modulates Light Responses in Horizontal Cells of the Turtle Retina
}

\author{
Abram Akopian ${ }^{1}$, John McReynolds ${ }^{2}$ and Reto Weiler ${ }^{1}$ \\ ${ }^{1}$ Department of Neurobiology, University of Oldenburg, PO Box 2503, 2900 Oldenburg, FRG \\ ${ }^{2}$ Department of Physiology, University of Michigan, Ann Arbor, MI 48109, USA
}

Key words: receptive field size, phorbol ester, dopamine, gap junctions

\begin{abstract}
The effect of phorbol esters on the light-evoked responses of horizontal cells were studied in the turtle eyecup preparation. Phorbol esters caused a reduction in receptive field size and a significant decrease in the amplitude of responses to annular and full-field illumination; however, they caused only minor changes in responses to small spots in the receptive field centre. The dark membrane potential was not affected. The results suggest that phorbol esters may affect both coupling resistance and membrane resistance in horizontal cells. The effects of phorbol esters were blocked by the protein kinase $\mathrm{C}$ inhibitor staurosporine, and inactive phorbol ester had no effect, making it very likely that the phorbol ester effects were mediated through activation of protein kinase $C$. The above effects of the phorbol esters were considerably reduced by the dopamine antagonists haloperidol and fluphenazine, suggesting that they were in part mediated by release of dopamine.
\end{abstract}

\section{Introduction}

Recent studies have suggested that protein kinase C (PKC) may be involved in some aspects of synaptic transmission between neurons (Nishizuka, 1984, 1986; Kaczmarek, 1986; Malinow et al., 1989). Immunocytochemical and biochemical studies have also revealed the presence of PKC in a variety of retinal neurons (Negishi et al., 1988; Cuenca et al., 1990; Greferath et al., 1990; Kato et al., 1990; Janssen and Weiler, 1991), but knowledge about the functional significance of its widespread distribution in the retina is still poor. Activation of PKC with phorbol esters facilitates the release of dopamine from the carp retina (Kato et al., 1990) and causes morphological changes in isolated horizontal cells from catfish retina (Rodrigues and Dowling, 1991). In the intact carp retina, activation of PKC with phorbol esters causes neurite formation; so-called spinules are formed on horizontal cell processes and invaginate into cone photoreceptor terminals (Weiler et al., 1991). The formation of these spinules also occurs during the process of light adaptation in the carp retina (Wagner, 1980; Weiler and Wagner, 1984), suggesting that activation of PKC may play a role in light adaptation.

Many retinal neurons, including horizontal cells, are electrically coupled through gap junctions (Kaneko, 1971; Vaney, 1991). The extensive coupling of these cells is subject to modulation depending on the ambient light conditions (Mangel and Dowling, 1985; Dong and McReynolds, 1991), a process which involves the neurotransmitter dopamine. Dopamine regulates a cAMP dependent protein kinase which in turn modulates junctional conductivity (Lasater, 1987; DeVries and Schwartz, 1989). In other tissues several other substances, including PKC, are known to affect gap junctional conductivity (Spray and Burt, 1990); activation of PKC increases the coupling resistance through phosphorylation at specific sites of the connexin protein (Asamota et al., 1991). The fact that several agents have been shown to regulate gap junction conductivity suggests that its regulation in retinal neurons may be complex and may also depend on the activation of PKC. However, it is not yet known how activation of PKC affects the light-evoked electrical responses of any neurons in the retina. The present study was undertaken to investigate possible effects of the activation of PKC within retinal neurons on the light-evoked responses of horizontal cells.

\section{Materials and methods}

Responses were recorded from horizontal cells in the inverted eyecup preparation of the red-eared turtle, Pseudemys scripta elegans. Animals were kept in a greenhouse under natural light conditions, and all experiments were performed in the afternoon. The preparation was made under dim room illumination. After decapitating the animal and removing the eye, the anterior portion of the eye was sliced away with a razor blade; after removing as much as possible of the vitreous humour with a paper wick, the eyecup was inverted and mounted with the convex, vitreous side up in the recording chamber, where it was

Correspondence to: Dr Reto Weiler, as above 
superfused with oxygenated turtle Ringer solution. The composition of the normal Ringer was (in $\mathrm{mM} / \mathrm{l}$ ): $\mathrm{NaCl} 110, \mathrm{KCl} 2.6, \mathrm{CaCl}_{2} 2.0$, $\mathrm{MgCl}_{2} 2.0$, glucose 10, HEPES buffer 5 . Known concentrations of test substances were added without substitution, and all solutions were adjusted to $\mathrm{pH} 7.4$ with $\mathrm{NaOH}$. PKC activity in retinal neurons was stimulated by adding the phorbol esters 12-O-tetradecanoyl-phorbol 13-acetate (TPA) or phorbol 12,13-dibutyrate (PDBu) to the superfusate, and PKC activity was inhibited by the addition of staurosporine. In some experiments the inactive phorbol ester 4-alphaphorbol 12,13-didecanoate was used. These agents were first dissolved in a small quantity of dimethyl sulphoxide and then diluted to the desired concentration in Ringer solution. Haloperidol and fluphenazine were dissolved in Ringer. TPA, PDBu and 4-alpha-phorbol 12,13-didecanoate were obtained from Serva, and staurosporine, haloperidol and fluphenazine from Sigma.

Intracellular recordings were made with conventional electronics and $3 \mathrm{M}$ potassium acetate-filled microelectrodes of 100-200 M resistance. Responses were stored on magnetic tape and displayed on a chart recorder or digital plotter. The results were obtained from luminosity-type horizontal cells, which were identified by their large receptive fields and large hyperpolarizing responses to light of all wavelengths. In most experiments the retina was kept in a light-adapted state by continuous exposure to a diffuse, steady, white background light, which caused a steady hyperpolarization of horizontal cells by about $5 \mathrm{mV}$. White light stimuli from a 100 -watt tungsten halogen source were presented as one of three standard test stimuli: a small spot ( $200 \mu \mathrm{m}$ diameter) centred in the cell's receptive field, a concentric annulus (1000 $\mu \mathrm{m}$ inside diameter, $2750 \mu \mathrm{m}$ outside diameter) and diffuse, full-field illumination of the entire retina. The unattenuated density flux at the plane of the retina was $10^{5} \mu \mathrm{watt} / \mathrm{cm}^{2}$. Figure legends indicate attenuation from this value.

\section{Results}

\section{Effects of phorbol esters on horizontal cell responses}

The phorbol esters TPA and PDBu caused a decrease in the amplitude of annulus and full-field responses in all cells, but they had variable effects on the responses to small, centred spots. In Figure 1, the three columns (A, B and C) show the effects of TPA in three different cells. In each column the responses in the top row are to the small spot stimulus, those in the middle row are to the annulus, and those in the bottom row are to full-field illumination. For each stimulus type a pair of responses is shown; the response on the left is a control and that on the right was recorded $5 \mathrm{~min}$ after the addition of $20 \mu \mathrm{M}$ TPA to the superfusate. These results are summarized in Figures 4 and 5 (unfilled bars). TPA $(1-20 \mu \mathrm{M})$ and PDBu $(2-10 \mu \mathrm{M})$ decreased the amplitude of the annular and full-field responses in all of the 43 cells tested; however, the response to the spot stimulus was increased in 13 cells, unchanged in 14 cells, and slightly reduced in 16 cells.

In a given experiment the type of effect on the spot response (increase, decrease or no change) persisted throughout the observation period (up to $60 \mathrm{~min}$ ) and was not related to the concentration of the phorbol ester. Statistical analysis of the data indicated that the observed variations did not represent three distinct groups. It is more likely that the different effects on the spot response represent variations in the extent to which the spot stimulated the peripheral portion of the receptive field. In most experiments there was no recovery of response amplitude when the superfusate was switched back to normal Ringer,

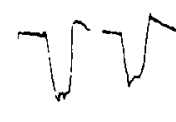

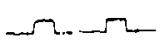
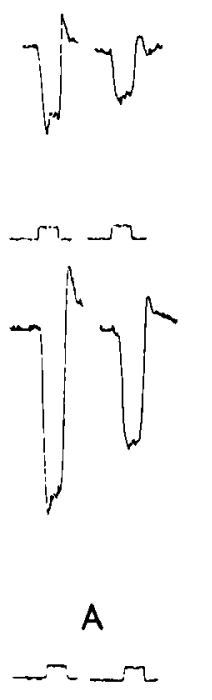
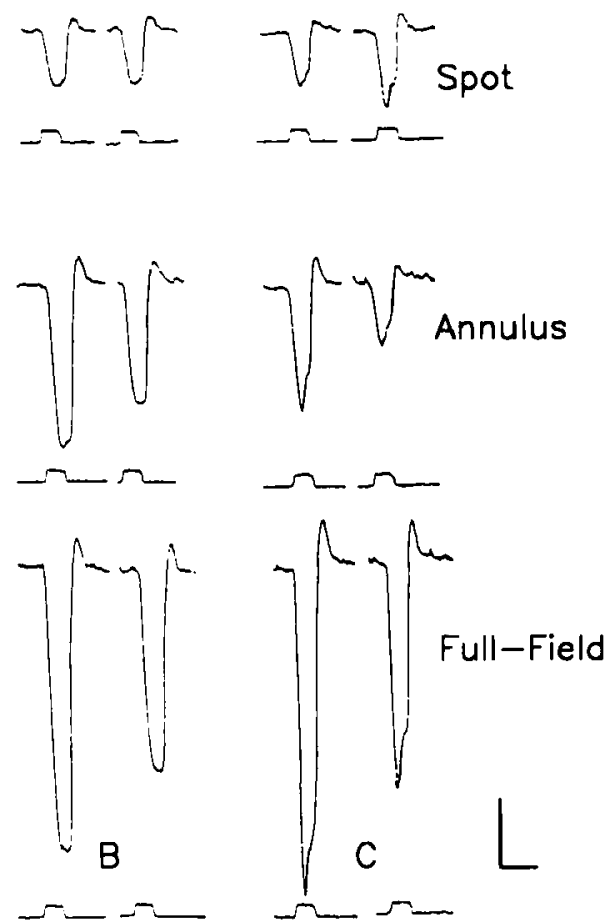

FiG. 1. Effect of TPA on horizontal cell responses to different stimulus patterns Columns A, B and C depict responses from three different cells. Upper, middle and lower rows show responses to centred spot, annulus and full-field stimuli. respectively. For each pair of responses, the one on the left was recorded in normal Ringer, and the one on the right after $5 \mathrm{~min}$ in $20 \mu \mathrm{M}$ TPA. Note the different effects of TPA on the spot responses in cells A, B and C. For all stimuli $\log 1=-1.5$. In this and all other experiments the diameter of the small spot was $200 \mu \mathrm{m}$, and the inside and outside diameters of the annulus were 1000 and $2750 \mu \mathrm{m}$, respectively. Calibration $10 \mathrm{mV}, 1 \mathrm{~s}$

even after $15 \mathrm{~min}$. The TPA-induced decrease in annulus and full-field responses was seen at all light intensities.

Figure 2 shows the effect of PDBu $(2 \mu \mathrm{M})$ on the horizontal cell response-intensity relationships. For the annular and full-field stimuli, but not the small spot stimulus, the response-intensity curves were markedly scaled down in amplitude, but the intensity which caused half-maximal responses was not changed. The effect of phorbol ester on receptive field size is shown in Figure 3; the plot shows the amplitudes of responses produced by a $200-\mu \mathrm{m}$ diameter spot at different displacements from the receptive field centre (Fig. 3A) and the amplitudes produced by spots of increasing diameter centred at the tip of the recording electrode (Fig. 3B). In the presence of phorbol ester (filled circles) the response amplitudes declined more rapidly with distance from the receptive field centre and large spots produced a much smaller response amplitude, indicating a reduction in the length constant of the horizontal cell network.

The inactive phorbol ester 4-alpha $(20 \mu \mathrm{M})$ had no effect in any of the four retinas tested, whereas subsequent addition of $20 \mu \mathrm{M}$ TPA had its typical effect in all of these retinas. Figure 4 shows that the effects of the phorbol esters were also greatly reduced in the presence of $2 \mu \mathrm{M}$ staurosporine, which inhibits the activation of PKC (Tamaoki et al., 1986). Thus, the above-decribed effects of TPA appear to be due to the activation of PKC. Staurosporine itself caused a slight 

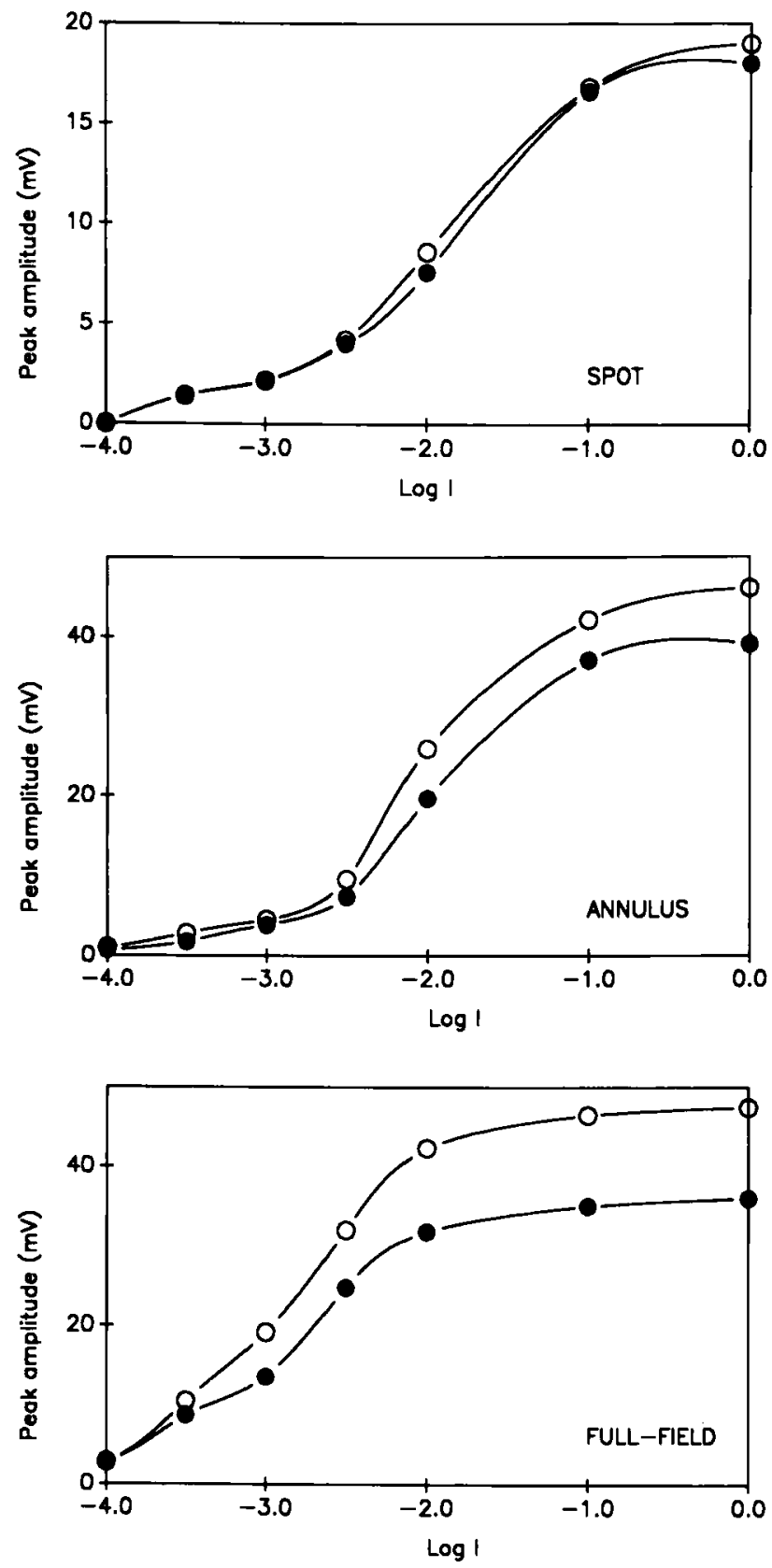

Fig. 2. Effect of PDBu on response-intensity relations. Open symbols. amplitude of control responses; filled symbols, response amplitude $10 \mathrm{~min}$ after addition of $2 \mu \mathrm{M}$ PDBu.

increase in amplitude of al! light responses, suggesting that there was some tonic level of PKC activation under the conditions of these experiments.

\section{Dopamine antagonists block the action of phorbol esters on horizontal cell responses}

Since phorbol esters have been shown to increase the release of dopamine in fish retina (Kato et al., 1990), some experiments were done to determine whether the above-described effects of phorbol esters
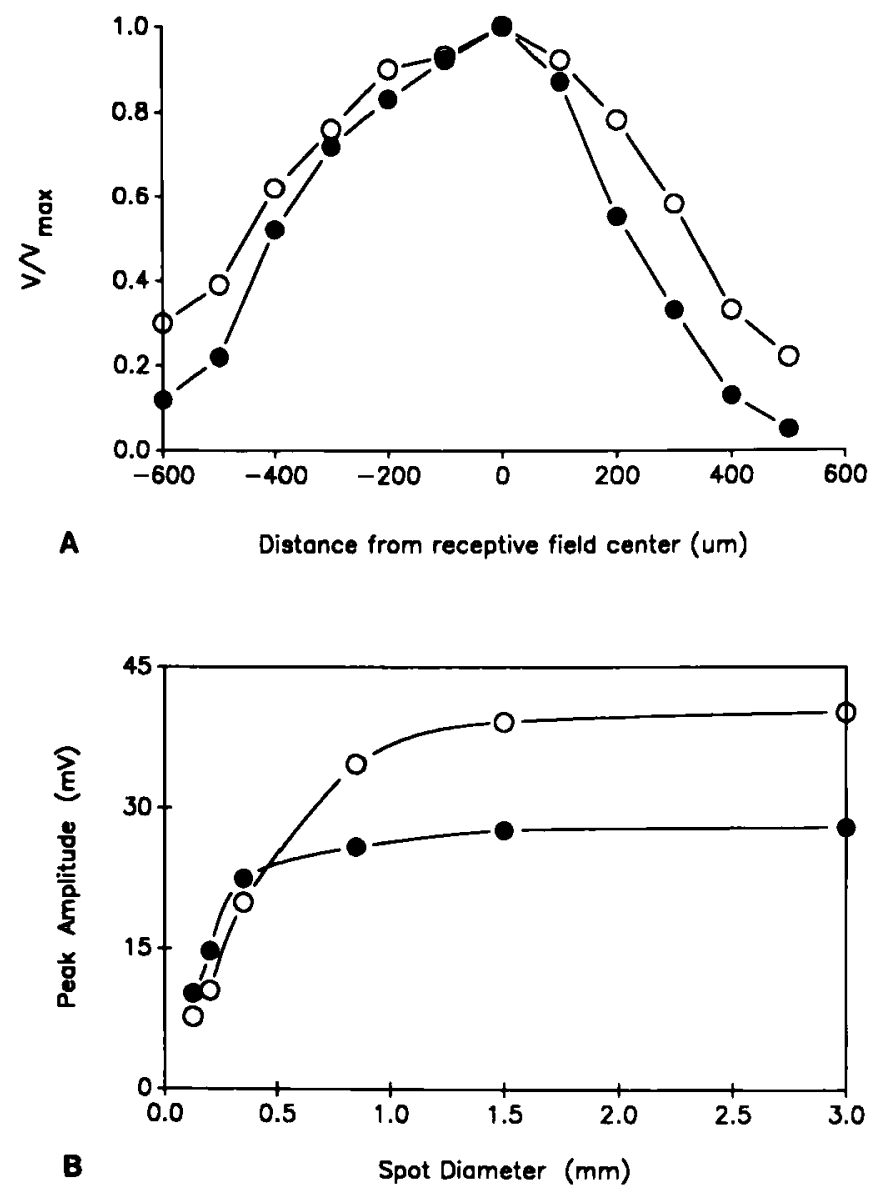

FIG. 3. Effect of TPA on receptive field profile. (A) Responses to $200-\mu \mathrm{m}$ diameter spot $(\log \mathrm{I}=-2.3)$ at different distances from receptive field centre. (B) Responses from another cell to spots of increasing diameter centred in the receptive field $(\log \mathrm{I}=-1.8)$. Open symbols, amplitude of control responses: filled symbols, response amplitude 8 min after addition of $20 \mu \mathrm{M}$ TPA.

on horizontal cell responses were due to an increase in dopamine release. Under our conditions the dopamine antagonists haloperidol and fluphenazine alone had no noticeable effect on horizontal cell responses, but the effects of the phorbol esters on horizontal cell responses were significantly reduced in the presence of dopamine antagonists. Figure 5A compares the effects of TPA in 27 control cells (unfilled bars) and in five cells in the presence of $100 \mu \mathrm{M}$ haloperidol (hatched bars); Figure 5B compares the effects of PDBu in 16 control cells (unfilled bars) and in four cells in the presence of $60 \mu \mathrm{M}$ fluphenazine (hatched bars). In the presence of either antagonist the phorbol esters had much less effect on annulus and full-field responses. Under these conditions the phorbol esters caused significant decreases in amplitude of all three types of light stimuli, but there was no evidence of a differential effect on spot and annulus responses.

\section{Discussion}

Phorbol esters always caused a large reduction in the amplitude of horizontal cell responses to full-field or annular illumination, but their effect on the responses to small, centred spot stimuli was variable (either 


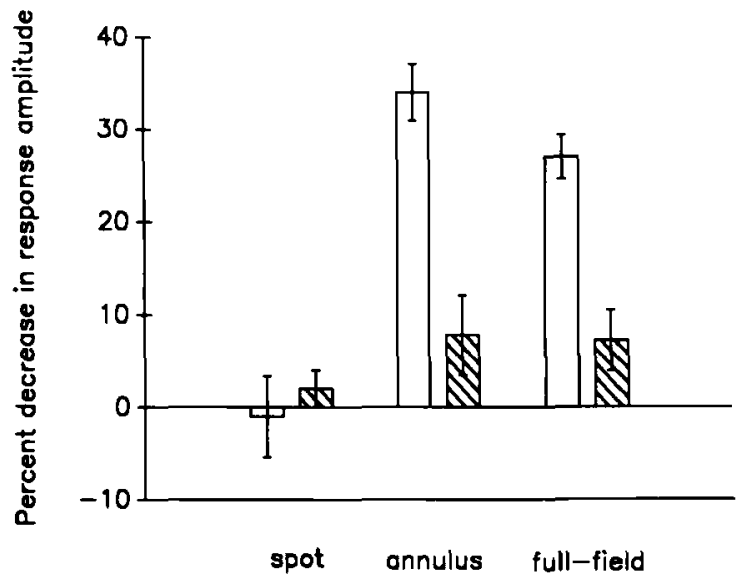

FIG. 4. Summary of effects of phorbol esters on horizontal cell responses to different light stimulus patterns in the presence and absence of staurosporine. Unfilled bars show per cent reduction in peak amplitude of light response (mean \pm SEM) to centred spot, annulus and full-field stimuli by phorbol esters $(10-20 \mu \mathrm{M}$ TPA or $2-10 \mu \mathrm{M}$ PDBu $)(n=43, \log \mathrm{I},-1.5$ to -2.0$)$. The phorbol esters reduced the amplitude of the annulus response by $34 \pm 3.1 \%$. and the full-field response by $27 \pm 2.4 \%$. This reduction was statistically significant $(P<0.01)$. The amplitude of the centred spot response was increased by $1.1 \pm 4.4 \%$; as noted above, this included increases in some cells and decreases in others. (The effects of TPA and PDBu are shown separately in Fig. 5.) Hatched bars, effect of the same concentrations of phorbol esters in the presence of $2 \mu \mathrm{M}$ staurosporine $(n=5, \log \mathrm{I}=-2)$. The effect of phorbo ester in the presence of staurosporine was significantly different $(P<0.05$, unpaired $t$-test) from its effect in the absence of staurosporine.

a small decrease or a small increase). Since the inactive phorbol ester 4-alpha-phorbol 12,13-didecanoate did not produce any of these effects, and since the effects were blocked by the PKC inhibitor staurosporine, it is likely that they were due to the activation of PKC. It is also likely that these effects are due to the activation of more than one type of PKC, as discussed below.

The site of the PKC activation on the retina which caused these changes in horizontal cell responses is not clear. PKC has been demonstrated immunocytochemically in cone pedicles, bipolar cells and some amacrine cells in turtle retina (Cuenca et al., 1990) and in some bipolar cells and dopaminergic cells in fish retina (Negishi $e t a l$. , 1988). Immunocytochemical studies have not revealed PKC in horizontal cells; however, there are several different subspecies of PKC and it is possible that horizontal cells contain a type of PKC which is not recognized by the antibodies used in those studies. In fact, other evidence suggests that some form of PKC is present in horizontal cells. For example, in fish phorbol esters induce anatomical changes in isolated horizontal cells (Rodrigues and Dowling, 1990) and cause phosphorylation of specific proteins in horizontal cell fractions (Janssen and Weiler, 1991). Also, the phorbol ester-induced spinule formation in fish horizontal cells was seen in retinas in which chemical synaptic transmission from other neurons was blocked (Weiler et al., 1991). The effects of phorbol esters on horizontal cell responses appear to be mediated by more than one mechanism; the present results suggest at least two actions which are mediated by an increase in dopamine release, as well as other actions which do not appear to be mediated by dopamine.

Two effects of phorbol esters on horizontal cell responses were greatly reduced in the presence of dopamine antagonists. One is

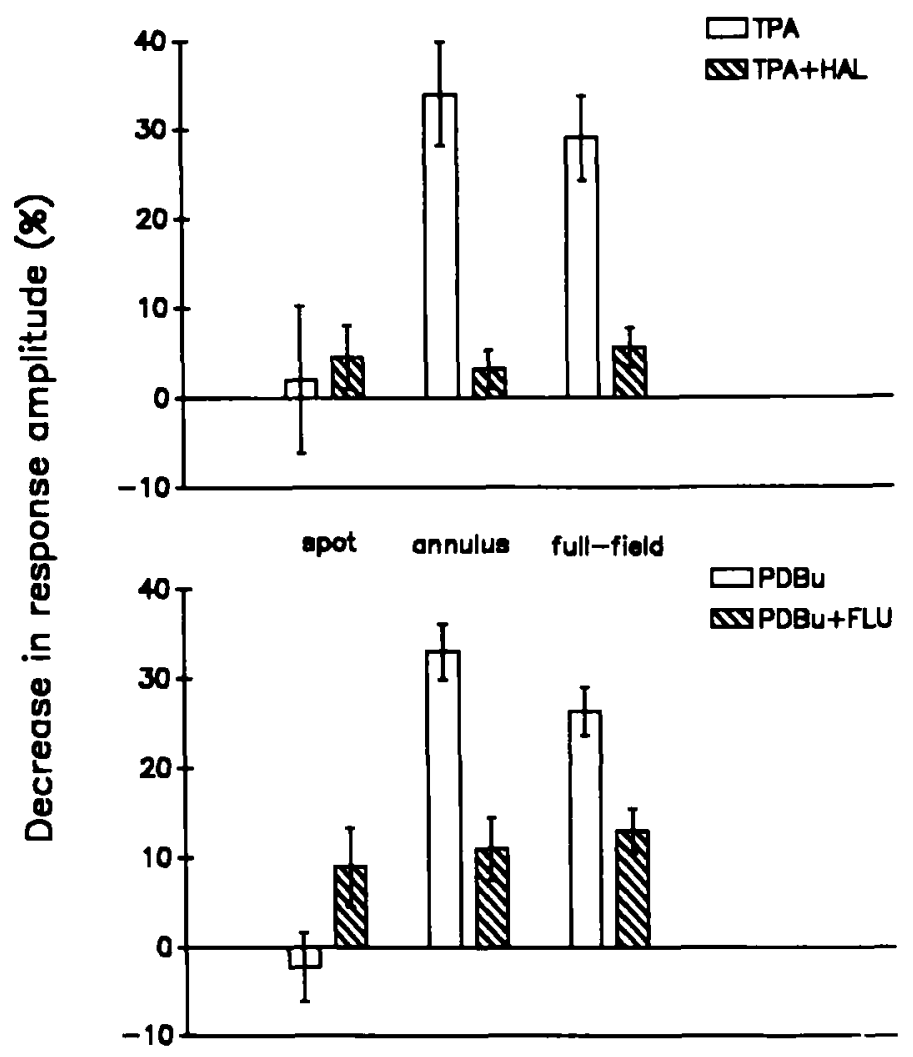

FIG. 5. Dopamine antagonists reduce the effects of phorbol esters on horizontal cell responses. Bars show per cent change in peak amplitude (mean \pm SEM) of light responses to small spot, annulus and full-field stimuli. Open bars show effect of phorbol esters alone; hatched bars show effect of phorbol ester in the presence of dopamine antagonist. (A) Phorbol ester was TPA $(10-20 \mu \mathrm{M})$ and antagonist was haloperidol (100 $\mu \mathrm{M})$. (B) Phorbol ester was PDBu $(2-20 \mu \mathrm{M})$ and antagonist was fluphenazine $(60 \mu \mathrm{M})$. The differences in $A$ and $B$ were statistically significant $(P<0.05$, unpaired $t$-test). Log I was -1.5 to -2.0 .

the increased response to small centred spots and reduction in annular response seen in some cells; this change is consistent with a dopamine-induced increase in coupling resistance between horizontal cells (Piccolino et al., 1984; Lasater and Dowling, 1985; Dong and McReynolds, 1991). The other is the decrease in full-field response amplitude; this effect cannot be explained by a change in coupling resistance since no lateral current flows between horizontal cells under full-field illumination, or by an increase in horizontal cell responsiveness to glutamate (Knapp and Dowling, 1987), since the latter would also cause a depolarization of horizontal cells in darkness, which was never observed in response to phorbol esters. The reduction in full-field responses thus appears to be due to some other action of dopamine. Phorbol esters cause the release of dopamine from interplexiform cells in fish (Kato et al., 1990), and it is possible that they could have a similar effect on the dopaminergic cells in turtle retina, which are believed to be amacrine rather than interplexiform cells (Witkovsky et al., 1984; Kolb et al., 1987).

The fact that some of the effects of phorbol esters were not completely blocked by dopamine antagonists, even at relatively high concentrations, suggests that activation of PKC may also have some other action(s) not related to an increase in dopamine release. The phorbol ester-induced spinule formation in fish horizontal cells is probably 
not due to an increase in dopamine release, since it was seen in retinas in which chemical transmission had been blocked with cobalt and also in retinas in which dopaminergic cells had been destroyed with 6-hydroxydopamine (Weiler et al., 1991). Although phorbol esters have been shown to reduce gap junction conductance in other tissues (reviewed in Spray and Burt, 1990), the fact that the major phorbol ester effect not blocked by dopamine antagonists was a decreae in responses to all light stimuli suggests that it was not a change in gap junction conductance. It is possible that the reduction in amplitude of horizontal cell responses was in part due to a reduction of light responses in photoreceptors, but this could not be tested in the present experiments due to difficulty in obtaining long-lasting recordings from photoreceptors.

Since PKC has been found in several different sites in the retina and may be present in all retinal cells, it is not surprising that its widespread activation by exogenously applied phorbol esters can cause complex physiological changes. Although phorbol esters clearly affect horizontal cell responses to light, these effects are difficult to explain by a single mechanism. It remains to be seen how the effects reported here are related to the light- and dark-adaptive changes mediated by dopamine and PKC activation (Mangel and Dowling, 1985; Kohler and Weiler, 1990; Weiler et al., 1991).

\section{Acknowledgements}

We thank Drs J. Ammermüller and W. Eldred for critical reading of the manuscript. Supported by the Deutsche Forschungsgemeinschaft and NIH Research Grant EY-01653.

\section{Abbreviations}

HEPES $\quad N$-(2-hydroxyethyl)piperazine- $N$ '-(2-ethanesulphonic acid)

$\mathrm{PDBu}$ phorbol 12,13-dibutyrate

PKC protein kinase $C$

TPA 12-O-tetradecanoyl-phorbol 13-acetate

\section{References}

Asamoto, M., Oyamada, M., El-Aoumari, A., Gros, D. and Yamasaki, H. (1991) Molecular mechanisms of TPA-mediated inhibition of gap-junctional intercellular communication: evidence for action on the assembly or function but not the expression of connexin 43 in rat liver epithelial cells. Mol. Carcinogenesis., 4, 322-327.

Cuenca, N., Fernandez, E. and Kolb, H. (1990) Distribution of immunoreactivity to protein kinase $C$ in the turtle retina. Brain Res., 532, 278-287.

DeVries, S. H. and Schwartz, E. H. (1989) Modulation of an electrical synapse between solitary pairs of catfish horizontal cells by dopamine and second messengers. J. Physiol. (Lond.), 414, 351-375.

Dong, C.-J. and McReynolds, J. S. (1991) The relationship between light, dopamine release and horizontal cell coupling in the mudpuppy retina. $J$. Physiol. (Lond.), 440, 291-309.

Greferath, U., Grünert, U. and Wässle, H. (1990) Rod bipolars in the mammalian retina show protein kinase C-like immunoreactivity. J. Comp. Neurol., 301, 433-442.

Janssen, U. and Weiler, R. (1991) PKC-mediated in vitro phosphorylation in isolated carp horizontal cells. Invest. Ophthalmol. Vis. Sci., 32 (Suppl.), 991.

Kaczmarek, L. K. (1986) Phorbol esters, protein phosphorylation and the regulation of neuronal ion channels. J. Exp. Biol., 124, 375-392.

Kaneko, A. (1971) Electrical connexions between horizontal cells in the dogfish retina. J. Physiol. (Lond.), 213, 95-105.

Kato, S., Ishita, S., Mawatari, K., Matsukawa, T. and Negishi, K. (1990) Dopamine release via protein kinase $\mathrm{C}$ activation in the fish retina. $J$. Neurochem., 54, 2082-2090.

Knapp, A. G. and Dowling, J. E. (1987) Dopamine enhances excitatory amino acid-gated conductances in cultured retinal horizontal cells. Nature, 325, 437-439.

Kohler, K. and Weiler, R. (1990) Dopaminergic modulation of transient neurite outgrowth from horizontal cells of the fish retina is not mediated by cAMP. Eur. J. Neurosci., 2, 788-794.

Kolb, H., Cline, C., Wang, H. H. and Brecha, N. (1987) Distribution and morphology of dopaminergic amacrine cells in the retina of the turtle (Pseudemys scripta elegans). J. Neurocytol., 16, 577-588.

Lasater, E. M. (1987) Retinal horizontal cell gap junction conductance is modulated by dopamine through a cyclic AMP-dependent protein kinase. Proc. Natl. Acad. Sci. USA, 84, 7319-7323.

Lasater, E. M. and Dowling, J. E. (1985) Dopamine decreases conductance of the electrical junctions between cultured retinal horizontal cells. Proc. Natl. Acad. Sci. USA, 82, 3025-3029.

Malinow, R., Schulman, H. and Tsien, R. W. (1989) Inhibition of post synaptic PKC or CaMKII blocks induction but not expression of LTP. Science, 245 , $862-866$

Mangel, S. C. and Dowling, J. E. (1985) Responsiveness and receptive field size of carp horizontal cells are reduced by prolonged darkness and dopamine. Science, 229, 1107-1109.

Negishi, K., Kato, S. and Teranishi, T. (1988) Dopamine cells and rod-bipolar cells contain protein kinase C-like immunoreactivity in some vertebrate retinas. Neurosci. Lett., 94, 247-252.

Nishizuka, Y. (1984) The role of protein kinase $C$ in cell surface signal transduction and tumour promotion. Nature, 308, 693-698.

Nishizuka, Y. (1986) Studies and perspectives of protein kinase C. Science, 233, 305-312.

Piccolino, M., Neyton, J. and Gerschenfeld, H. M. (1984) Decrease of gap junction permeability induced by dopamine and cyclic adeonsine $3^{\prime}: 5^{\prime}$-monophosphate in horizontal cells of turtle retina. J. Neurosci., 4, $2477-2488$.

Rodrigues, P. dos S. and Dowling, J. E. (1990) Dopamine induces neurite retraction in retinal horizontal cells via diacylglycerol and protein kinase $\mathrm{C}$. Proc. Natl. Acad. Sci. USA, 87, 9693-9697.

Spray, D. C and Burt, J. M. (1990) Structure - activity relations of the cardiac gap junction channel. Am. J. Physiol., 258, C195-C205.

Tamaoki, T., Nomoto, H., Takahashi, I., Kat, Y., Morimoto, M. and Tomita, F. (1986) Staurosporine, a potent inhibitor of phospholipid/Cadependent protein kinase. Biochem. Biophys. Res. Commun., 135, 397-402.

Vaney, D. I. (1991) Many diverse types of retinal neurons show tracer coupling when injected with biocytin or neurobiotin. Neurosci. Lett., 125, 187-190.

Wagner, H.-J. (1980) Light dependent plasticity of the morphology of horizontal cell terminals in cone pedicles of fish retinas. J. Neurocytol, , 9, 573-590.

Weiler, R. and Wagner, H.-J. (1984) Light-dependent change of cone-horizontal cell interactions in carp retina. Brain Res., 298, 1-9.

Weiler, R., Kohler, K. and Janssen, U. (1991) Protein kinase C mediates transient spinule-type neurite outgrowth in the retina during light adaptation. Proc. Natl. Acad. Sci. USA, 88, 3603-3607.

Witkovsky, P., Eldred, W. and Karten, H. J. (1984) Catecholamine- and indoleamine-containing neurons in the turtle retina. J. Comp. Neurol., 228 , $217-225$. 